\title{
A polarization dependent left handed metamaterial for telecommunication
}

\author{
Mehedi Hasan ${ }^{1 a)}$, Mohammad Rashed Iqbal Faruque ${ }^{1}$, \\ and Mohammad Tariqul Islam ${ }^{2}$ \\ ${ }^{1}$ Space Science Centre (ANGKASA), Universiti Kebangsaan Malaysia, Malaysia \\ 2 Department of Electrical, Electronic and Systems Engineering, \\ Universiti Kebangsaan Malaysia, Bangi 43600, Malaysia \\ a)mehedi.hasan.ukm@gmail.com,rashed@ukm.edu.my,tariqul@ukm.edu.my
}

\begin{abstract}
A polarization dependent, left handed metamaterial based on single layer patterned resonant structure intended for $\mathrm{C}$ - frequency band with $2.14 \mathrm{GHz}$ wide bandwidth is proposed. The size of a single unit cell is $11 \times 11 \mathrm{~mm}^{2}$ and the effective medium ratio is 5.27. CST Microwave Studio electromagnetic simulator is used to design and numerical analysis as well as Agilent N5227A VNA is for measurement. The measured results show resonance peaks at $5.17 \mathrm{GHz}$ and applicable for IEEE 802.11a Wi-Fi and cordless telephone applications. The proposed metamaterial is explained by lumped circuit model and exhibits left handed characteristics at $6.43 \mathrm{GHz}$.
\end{abstract}

Keywords: azimuthal angle, wide bandwidth, C-band, metamaterial Classification: Electromagnetic theory

\section{References}

[1] D. R. Smith, et al:: "Composite medium with simultaneously negative permeability and permittivity,” Phys. Rev. Lett. 84 (2000) 4184 (DOI: 10.1103/ PhysRevLett.84.4184).

[2] R. W. Ziolkowski: "Design, fabrication, and testing of double negative metamaterials,” IEEE Trans. Antennas Propag. 51 (2003) 1516 (DOI: 10.1109/ TAP.2003.813622).

[3] M. M. Hasan, et al:: "A new compact double-negative miniaturized metamaterial for wideband operation," Materials 9 (2016) 830 (DOI: 10. 3390/ma9100830).

[4] A. Sarkhel, et al.: "A compact meta-atom for dual band negative permittivity metamaterial,” Micro. Opti. Techn. Lett. 57 (2015) 1152 (DOI: 10.1002/mop. 29041).

[5] S. S. Islam, et al:: "The design and analysis of a novel split-H-shaped metamaterial for multi-band microwave applications," Materials 7 (2014) 4994 (DOI: 10.3390/ma7074994).

[6] H. Zhou, et al.: "A novel double-incidence and multi-band left-handed metamaterials composed of double Z-shaped structure," J. Mater. Sci.: Mater. Electron. 27 (2016) 2534 (DOI: 10.1007/s10854-015-4056-2).

[7] S. H. Liu, et al.: "Left-handed metamaterials based on only modified circular electric resonators," J. Mod. Opt. 63 (2016) 2220 (DOI: 10.1080/09500340. 2016.1189008).

[8] M. M. Hasan, et al:: "Composite left-handed meta-atom for tri-band operation," 
Materials Research Express 4 (2017) 095801 (DOI: 10.1088/2053-1591/ aa8824).

[9] S. S. Islam, et al.: "A new direct retrieval method of refractive index for metamaterials," Curr. Sci. 109 (2015) 337.

[10] M. M. Hasan, et al.: "A mirror shape chiral meta atom for C-band communication," IEEE Access 5 (2017) 21217 (DOI: 10.1109/ACCESS. 2017.2677463).

\section{Introduction}

Metamaterials are composed of periodic metals on dielectric materials have unique properties that are not available in the nature. Due to these unique properties, like negative refractive index, negative permeability, left handed characteristics, chirality, and so on, this artificial material has been received great attention in the physics and engineering communities. Metamaterial were first visionary speculated by the Russian Physicist Victor Veselago in 1967. After a long time, simultaneously negative permittivity and permeability were successfully invented by Smith et al. in 2000 [1]. Further, a metamaterial was developed in 2003 by R. W. Ziolkowski, which exhibited negative permittivity and negative permeability both at the X-band frequencies [2]. From the past age, different shape and structural metamaterials have been utilized for several applications, like, antenna design, electromagnetic absorption, invisible cloaking, etc. At present, for the rapid growth of modern telecommunication systems the demand of high performance, larger bandwidth and compactness have been increased exponentially. A compact z-shaped double negative metamaterial was exhibited for dual band application [3]. The dimension of the designed DNG metamaterial was $10 \times 10 \mathrm{~mm}^{2}$ and effective medium ratio (EMR) was 4.0. Sarkhel et al. exhibited a compact dual band "delta-shape" metamaterial applicable for C-band applications. The size of the metamaterial single unit cell was $7.40 \times 7.40 \mathrm{~mm}^{2}$ and the EMR was 7.50 in 2014 [4]. In addition, Islam et al. suggested a $30 \times 30-\mathrm{mm}^{2}$ 'H-shape' double negative metamaterial for multi-band applications and the resonance frequencies were in S-, C-, $\mathrm{X}$ - and $\mathrm{Ku}$-bands in 2014. In addition, the $\mathrm{H}$-shape metamaterial also explained by the open and interconnected array, but the EMR was only 3.64 [5]. In 2015, Zhou et al. proposed an $8.5 \times 8.5-\mathrm{mm}^{2}$ double negative metamaterial composed by coplanar electric and magnetic resonators, where the effective medium ratio was 4.80. A 'double Z-shape' metamaterial structure was composed of two orthogonal Z-shape metal strips resonator and exhibited resonance frequencies respectively at 7.3, 8.1, and 9.4 GHz [6]. In 2016, Liu et al. introduced a $5 \times 5-\mathrm{mm}^{2}$ "split-ringshape" dual-band metamaterial that was built by the modified circular resonators and exhibited left handed properties with the effective medium ratio was 5.45 [7]. In 2017, Hasan et al. shown a compact modified "I-shape" left handed meta-atom for S-, C-, and Ku-band applications with wide negative index bandwidth from 7.80 to $10.10 \mathrm{GHz}$ [8].

In this paper, a miniaturized left handed metamaterial that is composed by the 
cordless telephone applications, since the structure is applicable for C-band (5.15 to $5.35 \mathrm{GHz}$ ) application. The metamaterial is analyzed in different rotation angles; including $30^{\circ}, 45^{\circ}, 60^{\circ}$, and $75^{\circ}$ for relizaing effects of polarization angle of the incident wave on the performances. Table I shows the design specification of the proposed metamaterial single unit cell structure, where the substrate material length, width and height are respectively, 'a', 'b', and ' $t$ '. The metallic strips width (d) and height (h) are $0.50 \mathrm{~mm}$ and $0.035 \mathrm{~mm}$. The splits (s) and the length and width of the inner and outer ring resonators are respectively, '11', 'w1', 'l' and 'w'.

Table I. Design parameters of the metamaterial single unit cell.

\begin{tabular}{ccccccc}
\hline Parameters & $\mathrm{a}$ & $\mathrm{b}$ & $\mathrm{l}$ & $\mathrm{w}$ & 11 & $\mathrm{w} 1$ \\
\hline Dimensions $(\mathrm{mm})$ & 11 & 11 & 10 & 9.0 & 8.0 & 7.0 \\
\hline Parameters & $\mathrm{d}$ & $\mathrm{s}$ & $\mathrm{m}$ & $\mathrm{n}$ & 12 & $\mathrm{r} 2$ \\
\hline Dimensions $(\mathrm{mm})$ & 0.5 & 0.25 & 4.0 & 1.2 & 3.0 & 1.0 \\
\hline
\end{tabular}

Rogers RT 5880 dielectric material is used as a substrate material, which dielectric constant, loss tangent and thickness are respectively 2.20, 0.0009 and 1.575 $\mathrm{mm}$. However, the dimensions of the unit cell and array are shown in Fig. 1(a-c).

\section{Methodology}

The electromagnetic properties of the proposed metamaterial are characterized numerically by using finite integration technique based CST Microwave Studio package. This is a spatial discretization technique to solve electromagnetic field problems numerically in frequency domain. Basically, in this approach Maxwell's equations are applied in integral form to a set of staggered grids. Periodic boundary and perfect electromagnetic conductor boundary conditions are commonly used for the computation of metamaterial parameters. Periodic boundary is applied for simulating a part of mass framework by modeling its small part with the absence of proper surface. Perfect electromagnetic conductor boundary is the generalization of perfect electric and magnetic conductors as well as applied along $\mathrm{x}$ and y planes, so that electric field is oriented along $\mathrm{x}$-direction and magnetic field is oriented along y-direction shown in Fig. 2(a). The operating frequency ranges from 4 to $8 \mathrm{GHz}$, whereas the impedance is set to $50 \Omega$ and the EM-waves are incident on the designed metamaterial along the z-direction.

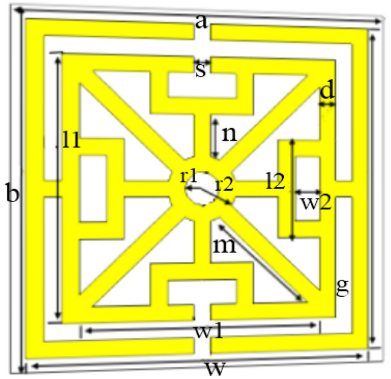

(a)

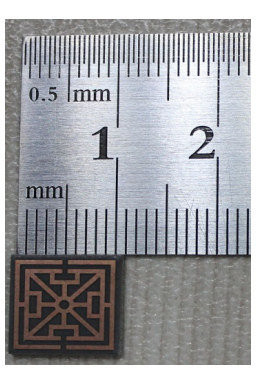

(b)

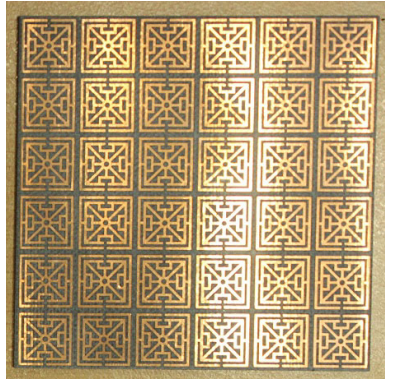

(c)

Fig. 1. Proposed Metamaterial: (a) Schematic structure, (b) Fabricated single unit cell structure, (c) Fabricated array structure.

The extraction of the effective medium parameters and normalized character- 


$$
\begin{gathered}
Z= \pm \sqrt{\frac{\left(1+S_{11}\right)^{2}+S_{21}^{2}}{\left(1-S_{11}\right)^{2}+S_{21}^{2}}} \\
\text { Permittivity, } \varepsilon_{e f f}=\left(\frac{\eta}{Z}\right) \\
\text { Permeability, } \mu_{\text {eff }}=(\eta \times Z) \\
n_{r}=\frac{c}{j \pi f d} \times\left\{\frac{\left(S_{21}-1\right)^{2}-S_{11}^{2}}{\left(S_{21}+1\right)^{2}-S_{11}^{2}}\right\}^{1 / 2}
\end{gathered}
$$

The resonance frequency $(f)$ of the proposed metamaterial is,

$$
f=\frac{1}{2 \pi \sqrt{L_{T} C_{T}}}
$$

The total inductance $\left(L_{T}\right)$ for the proposed metamaterial [10] can be obtained by,

$$
L_{T} \approx \mu_{0} t\left\{\frac{(a+b+s)^{2}+(m+n+d)^{2}}{\pi(l+w)\left(l_{1}+w_{1}\right)}+\frac{\sqrt{l_{1}^{2}+l_{2}^{2}+b^{2}}}{\sqrt{w^{2}+w_{1}^{2}+a^{2}}}\right\}
$$

The total capacitance $\left(C_{T}\right)$ can be calculated by,

$$
C_{T} \approx \varepsilon_{0} t\left[\frac{\sqrt{l^{2}+l_{1}^{2}+m^{2}}}{\sqrt{d^{2}+s^{2}}}-\ln \left\{\frac{(a+b)^{2}}{\pi\left(w_{1}^{2}-m^{2}-n^{2}\right)}\right\}\right]
$$

where, ' $d$ ' is thickness of substrate, ' $Z$ ' is normalize impedance, ' $k$ ' is wave vector, free space permeability ' $\mu_{0}$ ' is $4 \pi \times 10^{-7} \mathrm{H} / \mathrm{m}$ and permittivity ' $\varepsilon_{0}$ ' is $8.85 \times$ $10^{-12} \mathrm{~F} / \mathrm{m}$.

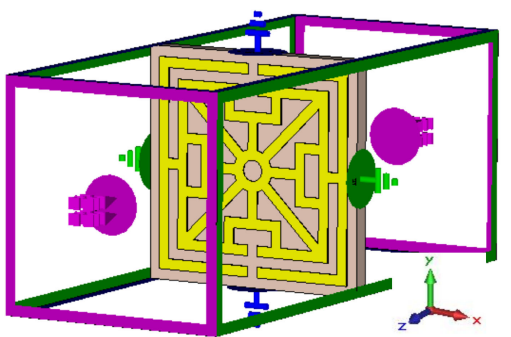

(a)

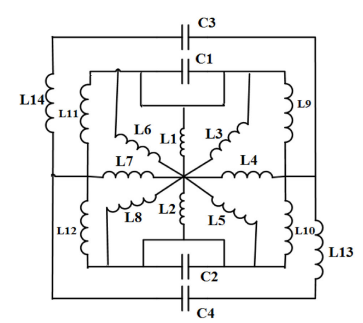

(b)

Fig. 2. (a) Boundary condition and simulated structure unit cell in the CST-Microwave Studio, (b) Lumped circuit model of the proposed metamaterial single unit cell.

In the above Fig. 2(b), for more split rings the number of gap and metallization on the substrate is increased. Besides, the increasing capacitance, which includes gap and surface capacitance, is reduced the operating frequency as they are inversely proportional to each other. A simple lumped element circuit is represented the analogy of the split ring resonator. The split rings form the magnetic metamaterial is considered as inductors and the capacitance is formed in and around gap areas. Moreover, the split ring resonator exhibits electromagnetic resonance, when the electric energy stored in capacitor; i.e., gap is in balance with the magnetic energy stored in the inductors.

\section{Results \& discussions}

An Agilent N5227A vector network analyser (VNA) is utilized to obtain the Sparameters as well as an Agilent N4694-60001 electronics calibration module is utilized to calibrate the N5227A-VNA for performing the measurement accurately. The long edges of the metamaterial is parallel to the electric field polarization and 
the electric response has been induced with the incident waves. In addition, loop is existed in the split ring resonators for inducing the magnetic response. From the surface current distribution in Fig. 3(a), it is realized that the lumped resonance is very stable and the magnetic field is dominant figure on the inner ring and the outer ring with capacitive gap area indicated in Fig. 3(b). The phase change of the proposed left handed are shown in Fig. 3(c).

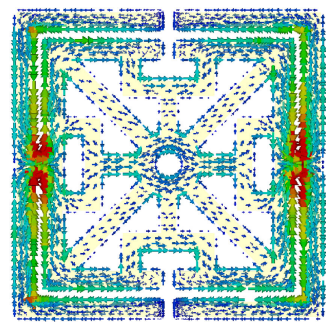

(a)

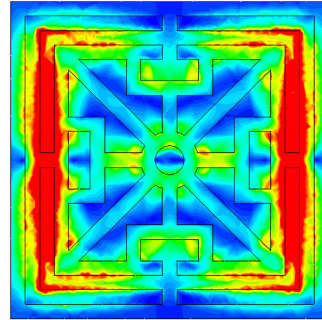

(b)

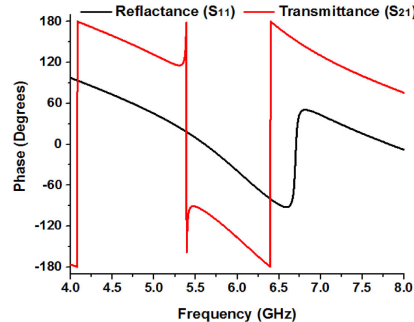

(c)

Fig. 3. (a) Surface current distribution, (b) Magnetic field, at $5.26 \mathrm{GHz}$; (c) Phase shift.

The measured transmittance shows the resonance at $5.17 \mathrm{GHz}$, which is in the C-band and the amplitude of the resonance point is $20.0 \mathrm{~dB}$, whereas the simulation transmittance at $5.26 \mathrm{GHz}$ and the magnitude of the resonance point is $43.0 \mathrm{~dB}$. In addition, the simulated reflectance is at $7.13 \mathrm{GHz}$, whereas the measured reflectance is at $6.98 \mathrm{GHz}$ in the same Fig. 4(a). Due to the fabrication process and measurement tolerance the results are slight discrepancy in Fig. 4(a). Moreover, the simulated and measured are showing the same frequency band for the linear polarization and can be altered by the parallel polarization.

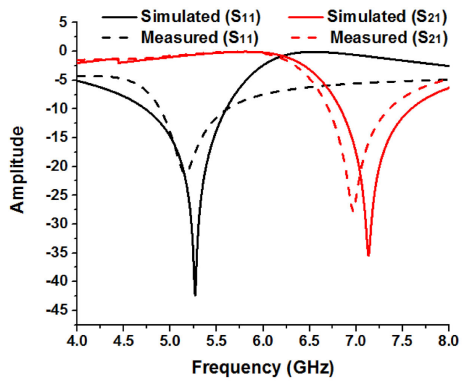

(a)

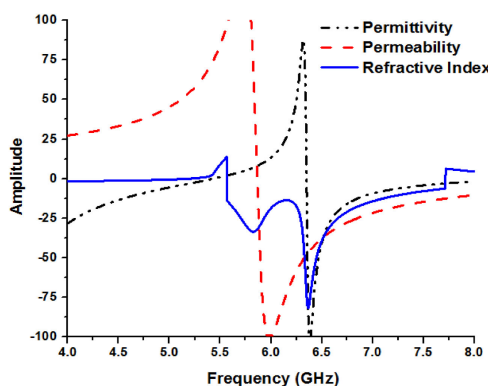

(b)

Fig. 4. (a) Transmittance $\left(\mathrm{S}_{21}\right)$ coefficient, (b) Magnitude of effective medium parameters of the proposed polarization dependent lefthanded metamaterial.

From Fig. 4(b), the negative permittivity from 4.0 to $5.44 \mathrm{GHz}$ and 6.35 to 8.0 GHz. Further, the permeability displays negative regime from 5.86 to $8.0 \mathrm{GHz}$. which covers bandwidth of $2.14 \mathrm{GHz}$. Moreover, the negative refractive index is from 4.0 to $5.22 \mathrm{GHz}$ and 5.57 to $7.72 \mathrm{GHz}$ in the same Fig. 4(b). Since, from 6.35 to $7.72 \mathrm{GHz}$ effective medium parameters are became negative. So, according to left handed characteristics the metamaterial is left handed metamaterial for any frequency point from 6.35 to $7.72 \mathrm{GHz}$. As a result, the backward waves from the designed metamaterial can be utilized to extend the range of beam scanning and the wave length are shorter than the intended EM-wave wavelengths. Besides, the transition region or stop band of the frequency bands are used to isolate or attenuate undesired EM-waves of the Wi-Fi and cordless telephone applications. 
Fig. 5(a) represents the effect on transmittance $\left(\mathrm{S}_{21}\right)$ through different dielectric materials using as a substrate material. The dielectric constant of the FR-4, Rogers RT 5880, Rogers RT 5870, Rogers RT 6010, Rogers RT 4350, Rogers RT 4003, Mica and Polyimide are respectively 4.50, 2.20, 2.35, 10.70, 3.66, 3.55, 5.70 and 3.50. Due to the raise of dielectric constant the materials conductivity is decreased. The permittivity is depend on material internal structure, where the electric and magnetic fields are oscillating as sinusoidal pattern as well as the atomic velocity depend on the conductivity. In addition, increasing the dielectric constant causes an increase for capacitance between the ground plane and resonator.

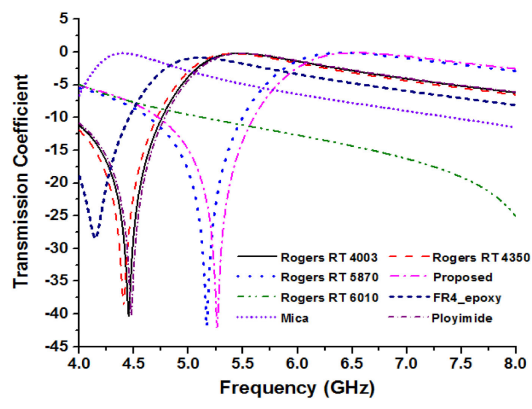

(a)

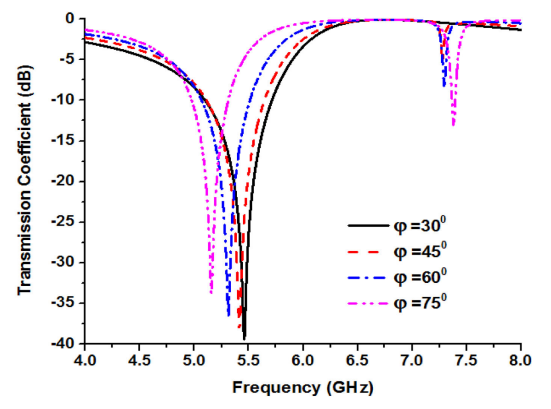

(b)

Fig. 5. Transmittance $\left(S_{21}\right)$ at different: (a) Dielectric materials, (b) Angular rotations.

In Fig. 5(b), at $30^{\circ}, 45^{\circ}, 65^{\circ}$ and $75^{\circ}$ azimuthal $(\varphi)$ angular rotations the resonance frequencies are respectively, at 5.45, 5.41, 5.31 and $5.15 \mathrm{GHz}$. When the incidence angle increases for TM incidence, the strength of the incident H-field parallel to the y-direction grating groove and does not change. The strength of the oscillating current loop for magnetic polarization (MP) does not decrease, as well as the MP resonance strength remains almost unchanged. Contrary, for TE-waves the transmittance at MP resonance increases slightly with larger oblique angles because of the H-field component parallel to the grating groove is decreased. Table II shows, proposed metamaterial bandwidth (BW) is triple and seventh than the refs. [5], [6] as well as EMR is more than refs. [5, 6] and similar to the ref. [7].

Table II. Comparisons between the proposed metamaterial (MM) and previous researches

\begin{tabular}{|cccccc|}
\hline References & Year & Dimensions & Freq. Band & BW $(\mathrm{GHz})$ & EMR $(\lambda / a)$ \\
\hline Islam et al. [5] & 2014 & $30 \times 30 \mathrm{~mm}^{2}$ & Multi Band & 0.90 & 3.64 \\
\hline Zhou et al. $[6]$ & 2015 & $8.5 \times 8.5 \mathrm{~mm}^{2}$ & X-Band & 0.30 & 4.80 \\
\hline Liu et al. [7] & 2016 & $5.0 \times 5.0 \mathrm{~mm}^{2}$ & X-Band & 1.20 & 5.45 \\
\hline Proposed MM & 2017 & $11 \times 11 \mathrm{~mm}^{2}$ & C-Band & 2.15 & 5.27 \\
\hline
\end{tabular}

\section{Conclusion}

A compact metamaterial printed on Rogers RT 5880 dielectric material is reported; that exhibits resonance at $5.17 \mathrm{GHz}$ and a bandwidth of $2.15 \mathrm{GHz}$. The simulated and experimental results show excellent agreement, suggesting the metamaterial performs very well in C-band for IEEE 802.11a Wi-Fi and cordless telephone communication. The effects of substrate material on resonance frequency is also investigated, whereas the permittivity of the substrate material is inversely proportional with the resonance peaks of the proposed metamaterial. 